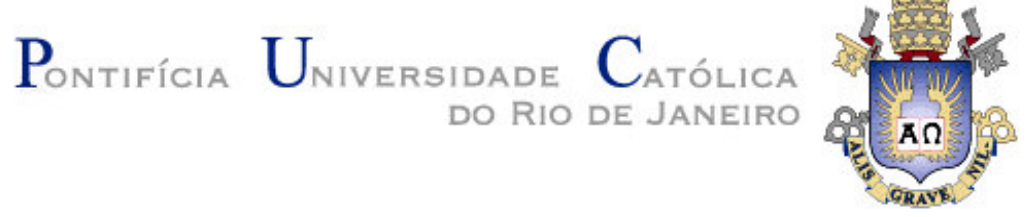

João Vicente Falabella Fabrício

\title{
Análises Probabilísticas da Estabilidade de Taludes e Contenções
}

Dissertação de Mestrado

Dissertação apresentada como requisito parcial para obtenção do título de Mestre pelo Programa de PósGraduação em Engenharia Civil da PUC-Rio.

Orientadores: Prof. Alberto S. F. Jardim Sayão Prof Paulo César de Almeida Maia 


\section{Análises Probabilísticas da Estabilidade de Taludes e Contenções}

Dissertação apresentada como requisito parcial para obtenção do título de Mestre pelo Programa de PósGraduação em Engenharia Civil da PUC-Rio. Aprovada pela Comissão Examinadora abaixo assinada.

Prof. Alberto S. F. Jardim Sayão Orientador Puc - Rio

Prof. Paulo César de Almeida Maia Co-orientador UENF

Prof. Anna Laura Lopes da Silva Nunes COPPE

Prof. Sérgio Augusto B. Fontoura

Puc - Rio

Prof. Willy de Alvarenga Lacerda COPPE

Prof. José Eugênio Leal Coordenador Setorial do Centro Técnico Científico - PUC-Rio 
Todos os direitos reservados. É proibida a reprodução total ou parcial do trabalho sem autorização da universidade, do autor e do orientador.

João Vicente Falabella Fabrício

Graduou-se em Engenharia Civil, pela Pontifícia Universidade Católica do Rio de Janeiro em 1986. Pós-graduação Executiva em Meio Ambiente COPPEUFRJ no ano de 2001. Coordenou e atuou em inúmeros projetos fiscalização de implantações e restaurações de pavimentos rodoviários e urbanos. Participou das pesquisas "Monitoramento de Variáveis Geoambientais com o Emprego de Imagens Orbitais" e "Estabelecimento para critérios de qualidade para agregados pétreos" contratadas pelo Instituto de Pesquisa Rodoviárias respectivamente em 1992 e 1998.

Ficha Catalográfica

Fabrício, João Vicente Falabella

Análises probabilísticas da estabilidade de taludes e contenções / João Vicente Falabella Fabrício ; orientadores: Alberto S. f. Jardim Sayão, Paulo César de Almeida Maia. - Rio de Janeiro : PUC, Departamento de Engenharia Civil, 2006.

170 f. : il. ; $30 \mathrm{~cm}$

Dissertação (mestrado) - Pontifícia Universidade Católica do Rio de Janeiro, Departamento de Engenharia Civil.

Inclui referências bibliográficas.

1. Engenharia civil - Teses. 2. Estatística. 3. Análises Determinísticas de Ruptura de Taludes e Muro de Contenção. 4. Análises Probabilísticas da Estabilidade de Taludes eCcontenções. I. Sayão, Alberto S. F. Jardim. II. Maia, Paulo César de Almeida. III. Pontifícia Universidade Católica do Rio de Janeiro. Departamento de Engenharia Civil. IV. Título. 


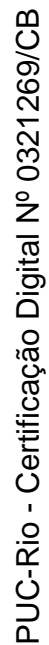

A todos aqueles que direta ou indiretamente contribuíram para este trabalho. 


\section{Agradecimentos}

Ao Prof. Sayão, que com seu vasto conhecimento técnico proporcionou um excelente respaldo para a conclusão desse trabalho.

Ao Prof. Paulo Maia, que pela competência, amizade e dedicação teve grande importância nessa pesquisa.

À minha mulher pela ajuda prestada. 


\section{Resumo}

Fabrício, João Vicente Falabella; Sayão, Alberto S. F. Jardim; Maia, Paulo César de Almeida. Análises probabilísticas da estabilidade de taludes e contenções. Rio de Janeiro, 2006. 170p. Dissertação de Mestrado - Departamento de Engenharia Civil, Pontifícia Universidade Católica do Rio de Janeiro.

Os projetos geotécnicos são, em geral, avaliados através do cálculo de um coeficiente de segurança, obtido de forma determinística. A presente pesquisa tem como objetivo a comparação de dois métodos probabilísticos (Estimativas Pontuais e Segundo Momento) empregados em análises de estabilidade. Os métodos probabilísticos quantificam as incertezas oriundas da variabilidade dos parâmetros geotécnicos, calculando-se um índice de confiabilidade $(\beta)$, que expressa o quanto o fator de segurança é confiável. No entanto, o índice $\beta$ é um parâmetro relativo, pois não exprime todas as incertezas contidas em um projeto geotécnico. O trabalho apresenta uma comparação das análises probabilísticas de estabilidade da barragem de Curuá-Una, no Pará, e de um muro de arrimo, em São Fidélis, no Estado do Rio de Janeiro. No caso da barragem, estudou-se a influência de um eventual aumento no nível d'água do reservatório no cálculo da probabilidade de ruptura. Para o muro, foram consideradas diversas alturas do nível d'água no retroaterro a montante. As análises enfocaram, ainda, detalhes dos métodos probabilísticos, no que se refere à aplicabilidade para projetos práticos. Verificou-se que o método do Segundo Momento é de mais fácil utilização em geotecnia. Recomenda-se, portanto, a sua aplicação em conjunto com as análises determinísticas, mais usuais na prática.

\section{Palavras-chave}

Métodos probabilísticos; Segundo Momento; Análises determinísticas. 


\section{Abstract}

Fabrício, João Vicente Falabella; Sayão, Alberto S. F. Jardim (Advisor); Maia, Paulo César de Almeida (Advisor). Probabilistic analyses of slopes and retainments stability. 170p. Rio de Janeiro, 2006. MSc. Dissertation - Departamen of Civi Engineering, Pontifícia Universidade Católica do Rio de Janeiro

Geotechnical projects are generaly assessed through the calculus of the safety factor obtained by deterministic methodologies. The purpose of the present research work is to compare two probabilistic methods (Estimate Punctual and Second Moment) employed in stability analyses. Probabilistic methods quantify the uncertainties derived from the variability of geotechnical parameters, calculating a confidence ratio that expresses how reliable the safety factor is. However, the ratio is a relative parameter, since it does not account for all the uncertainties contained in a geotechnical project. In view of this situation, the study compares the stability probabilistic analyses of Curuá-Una dam, in Pará, and those of a retaining wall in São Fidélis, Rio de Janeiro. Regarding the dam, the influence of a possible rise in the resevoir water level in the calculus of failure probability has been investigated. In respect to the wall, several water levels have been considered in the upstream backfill. Moreover, the analyses have focused on the details of probabilistic methods and their application to practical projects. It has been verified that the Second Moment Method is more easily employed in geothecnics. Therefore, it is recommended that it should be applied together with the deterministic analyses currently used in practice.

\section{Keywords}

Probabilistic methods; Second Moment; deterministic analyses. 


\section{Sumário}

1 Introdução 17

2 Análises Determinísticas de Estabilidade 20

2.1 Introdução 20

2.2 Métodos Determinísticos de estabilidade de taludes 20

2.2.1 Hipóteses Básicas Consideradas nos Métodos de Equilíbrio Limite 21

2.2.2 Método das Fatias 21

2.2.3 Método de Fellenius (1927) 22

2.2.4 Método de Bishop Simplificado (1955) 24

2.2.5 Método de Janbu Simplificado (1955) 25

2.2.6 Método de Spencer (1967) 26

2.2.7 Método de Morgenstern \& Price (1965) 29

2.2.8 Método de Sarma (1973) 30

2.2.9 Comentários 30

2.3 Empuxo de Terra 31

2.3.1 Teoria de Rankine (1857) 31

2.3.2 Teoria de Coulomb (1776) 34

2.3.3 Métodos das Cunhas $\quad 35$

2.4 Análise Determinística de um Muro de Contenção 36

2.41 Segurança contra o Tombamento 36

$\begin{array}{ll}2.42 \text { Segurança contra o Escorregamento } & 37\end{array}$

2.4.3 Segurança contra a Ruptura e Deformação Excessiva do Terreno de Fundação 38

2.4.4 Segurança Contra a Ruptura do Conjunto Solo/Muro 39

3 Análises Probabilísticas de Estabilidade $\quad 40$

3.1 Introdução 40

3.2 Conceitos de Estatística 40 
3.2.1 Definições Gerais $\quad 40$

3.2.2 Tendência Central 41

3.2.3 Medidas de Dispersão 42

3.2.4 Distribuição da Probabilidade dos Parâmetros Geotécnicos 44

3.2.5 Distribuição Normal $\quad 45$

3.2.6 Distribuição Log-Normal $\quad 47$

3.2.7 Estimativa de Intervalos de Confiança $\quad 47$

3.3 Tipos de Análises Probabilísticas $\quad 50$

3.4 Formulação do Índice de Confiabilidade e da Probabilidade de Ruptura 51

3.5 Métodos Probabilísticos 55

3.5.1 Simulação de Monte Carlo $\quad 55$

3.5.2 Método das Estimativas Pontuais 56

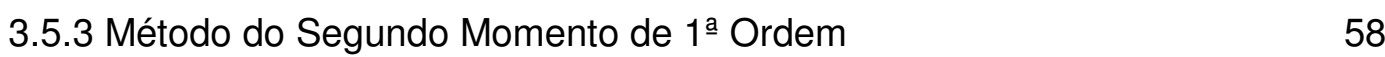

3.6 Parâmetros para Análise Probabilística 62

3.6.1 Fontes de Incertezas $\quad 62$

3.6.2 Erros Sistemáticos $\quad 62$

3.6.3 Dispersão de Dados 63

3.7 Número Mínimo de Ensaios 63

3.8 Cálculo dos Momentos Probabilísticos do Ângulo de Atrito e da Coesão 66

3.8.1 Ensaio de Cisalhamento Direto 66

$\begin{array}{ll}\text { 3.8.2 Ensaio Triaxial } & 67\end{array}$

$\begin{array}{ll}3.9 \text { Considerações Finais } & 67\end{array}$

4 Análise Probabilística de Ruptura da Barragem de Curuá-Una para Diversos Níveis do Reservatório. $\quad 69$

$\begin{array}{ll}4.1 \text { Localização } & 69\end{array}$

4.2 Geometria, Parâmetros Geotécnicos e Posicionamento

da Linha Freática. $\quad 70$

4.3 Estudos Probabilísticos 73

$\begin{array}{ll}\text { 4.3.1 Programa Determinístico } & 73\end{array}$

$\begin{array}{ll}\text { 4.3.1.1 Dados de Entrada } & 74\end{array}$

$\begin{array}{ll}\text { 4.3.1.2 Dados de Saída } & 74\end{array}$

4.3.2 Procedimentos e Resultados $\quad 74$

$\begin{array}{ll}4.4 \text { Análises dos Resultados } & 78\end{array}$

$\begin{array}{ll}\text { 4.4.1 Números de Variáveis } & 78\end{array}$

4.4.2 Fixação ou Não da Superfície de Ruptura 78

4.4.3 Comparação Entre os Métodos 80 
5 Análise Probabilística de Um Muro de Contenção 82

5.1 Introdução 82

5.2 Localização Geográfica

5.3 Perfil do Talude $\quad 82$

5.4 Parâmetros dos Solos $\quad 84$

$\begin{array}{ll}5.5 \text { Programa Utilizado } & 87\end{array}$

5.5.1 Introdução $\quad 87$

$\begin{array}{ll}\text { 5.5.2 Dados de Entrada } & 87\end{array}$

5.5.2.1 Dados Geométricos em Relação à Geometria do Muro 87

5.5.2.2 Dados em Relação a Geometria do Solo e Suas Propriedades 87

5.2.2.3 Dados em Relação ao Nível D’água e as Cargas Atuantes 89

5.5.3 Cálculo do Empuxo $\quad 89$

5.5.4 Dados de Saída 90

$\begin{array}{ll}5.6 \text { Análises Probabilísticas } & 90\end{array}$

5.6.1 Geometria do Muro de contenção 9

$\begin{array}{ll}\text { 5.6.2 Parâmetros de Entrada } & 91\end{array}$

5.6.3 Análise de Sensibilidade 92

5.7 Análise dos Resultados $\quad 95$

5.8 Comentários 102

6 Conclusões 106

$\begin{array}{ll}\text { 6.1 Barragem de Curuá-Una } & 106\end{array}$

6.1.1Fixação ou Não da Superfície de Ruptura 106

6.1.1 Comparação Entre os Métodos 106

$\begin{array}{ll}\text { 6.2 Muro de Contenção } & 106\end{array}$

6.3 Conclusões Gerais 107

$\begin{array}{ll}\text { 6.4 Sugestões para Futuras Pesquisas } & 108\end{array}$

7 Referências Bibliográficas $\quad 109$

$\begin{array}{ll}\text { Anexo } 1-\text { Tabelas estatísticas } & 113\end{array}$

Anexo 2 - Tabelas de cálculo do Capítulo $4 \quad 115$

Anexo 3 - Tabelas de cálculo do Capítulo 5 


\section{Lista de Figuras}

Figura 2.1 - Forças atuantes em uma fatia genérica 22

Figura 2.2 - Forças atuantes em uma fatia 23

Figura 2.3 - Fator $f_{0}$ em função do parâmetro d/L 26

Figura 2.4 - Forças atuantes na base da fatia $\quad 27$

Figura 2.5 - Fator de segurança que satisfaz as equações 2.13 e $2.14 \quad 28$

Figura 2.6 - Forças atuantes em uma fatia $\quad 29$

Figura 2.7 - Empuxo de terra em um muro contendo um terreno com inclinação $\beta$ em relação a horizontal 32

Figura 2.8 - Distribuição das tensões horizontais em um solo coesivo 33

Figura 2.9 - Forças atuantes em uma possível cunha de ruptura. 34

Figura 2.10 - Equilíbrio das forças que atuam na cunha de ruptura 35

Figura 2.11 - Método das cunhas 36

$\begin{array}{ll}\text { Figura 2.12 - Forças atuantes em um muro de peso } & 37\end{array}$

Figura 2.13 - Distribuição de pressões no solo 38

Figura 2.14 - Distribuição triangular de pressões no solo 39

Figura 2.15 - Formas de instabilidade em um muro de contenção 39

Figura 3.1 - Distribuição estatística das médias das amostras

da população densidade natural do solo 41

Figura 3.2 - Distribuição estatística das médias das amostras

da população coesão do solo $\quad 43$

Figura 3.3 - Comparação das dispersões entre as distribuições $\bar{X}_{i}$ e $\bar{Y}_{i} \quad 43$

Figura 3.4-Gráfico de uma distribuição normal 45

Figura 3.5 - Transformação linear da curva normal geral

em curva normal padrão 46

Figura 3.6 - Gráfico de X x f(x) de uma distribuição log -normal 47

Figura 3.7- Comparação entre a curva normal e as curvas de Student 48

Figura 3.8 - Curvas de distribuição de probabilidade do Fator de Segurança e a curva de Gauss normalizada $\quad 52$

Figura 3.9 - Confiabilidade x Probabilidade de Ruptura 53

Figura 3.10 - Aumento do $\bar{F} \bar{S}$ mantendo-se constante $\sigma_{F S}$ 
Figura 3.11 - Aumento do $\sigma_{F S}$ mantendo-se $\bar{F} \bar{S}$ constante

Figura 3.12- Distribuição probabilística de duas curvas com desvios padrões diferentes $\quad 54$

Figura 3.13 - Ângulo de Atrito x FS 61

Figura 3.14 - Densidade x FS 61

Figura 3.15 - Gráfico da Acurácia x Número de Ensaios 65

Figura 4.1 - Localização da Barragem de Curuá-Uma 69

Figura 4.2 - Desenho da Barragem de Coruá-Uma em escala deformada $\quad 70$

Figura 4.3 - Posicionamento dos piezômetros ao longo da estaca 35

Figura 4.4 -Cota Piezométrica Para o Nível do Reservatório Igual a 67 m 73

Figura 4.5 - Influências dos parâmetros geotécnicos na variância

do FS para o caso do NA igual a 67,0m e mantendo-se a superfície $\begin{array}{ll}\text { de ruptura fixa } & 75\end{array}$

Figura 4.6 - Fator de segurança em função do nível do reservatório 76

Figura 4.7 - Comparação do método do Segundo Momento

de Primeira Ordem utilizando 3 variáveis e 18 variáveis

Figura 4.8 - Comparação entre os métodos do Segundo Momento

e das Estimativas Pontuais utilizando para ambos 3 parâmetros

e; fixando e deixando móvel a superfície de ruptura

Figura 4.9 - Razão da probabilidade de ruptura entre os métodos

do Segundo Momento e das Estimativas Pontuais utilizando, para ambos, 3 parâmetros e fixando a superfície de ruptura.

Figura 5.1 - Levantamento topográfico planialtimétrico e localização

da área de estudo

83

Figura 5.2 - Seção crítica da área de estudo e o perfil geológico 84

Figura 5.3 - Dados da geometria do muro 88

Figura 5.4 - Dados geométricos em relação ao solo 88

Figura 5.5 posição do níveld'água $\quad 89$

Figura 5.6 - Valor do empuxo em relação ao ângulo

da possível cunha de ruptura 90

Figura 5.7 - Geometria do muro de contenção usado

na análise probabilística $\quad 92$

Figura 5.8 - Análise de sensibilidade para o tombamento 93

Figura 5.9- Análise de sensibilidade para o deslizamento 93

Figura 5.10 - Análise de sensibilidade para o terreno de fundação 94

Figura 5.11 - Variação do fator de segurança contra

o tombamento com a largura da base do muro e o nível d’água. 
Figura 5.12 - Fator de segurança contra o deslizamento x largura

da base do muro x nível d'água

Figura 5.13 - Fator de segurança contra a ruptura do solo de fundação x largura da base do muro x nível d'água.

Figura 5.14 - Confiabilidade contra o tombamento $x$ largura

da base do muro $x$ nível d'água obtida através do

método do Segundo Momento.

Figura 5.15 - Confiabilidade contra o deslizamento x largura

da base do muro x nível d'água obtida através do método

do Segundo Momento de Primeira Ordem

Figura 5.16 - Confiabilidade contra a ruptura do solo de fundação x largura da base do muro x nível d'água obtida através do método do Segundo Momento

Figura 5.17 - Confiabilidade contra o tombamento $x$ largura da base do muro $x$ nível d'água obtida através do método das Estimativas Pontuais

Figura 5.18 - Confiabilidade contra o deslizamento x largura da base do muro x nível d'água obtida através do método das Estimativas Pontuais

Figura 5.19 - Confiabilidade contra a ruptura do solo de fundação x largura da base do muro x nível d'água obtida através do método das Estimativas Pontuais

Figura 5.20 - Variância do Fator de Segurança contra o

tombamento $\mathrm{x}$ largura da base do muro $\mathrm{x}$ nível d'água obtida através do método do Segundo Momento

Figura 5.21 - Variância do Fator de Segurança contra o deslizamento x largura da base do muro x nível d'água obtida através do método do Segundo Momento.

Figura 5.22 - Variância do Fator de Segurança contra a ruptura do solo de fundação x largura da base do muro x nível d'água obtida através do método do Segundo Momento. 


\section{Lista de Tabelas}

Tabela 3.1 - Número de simulações necessárias para manter uma precisão $(\varepsilon \%)$, com um nível de confiança $(1-\alpha)$, considerando $\mathrm{n}$ variáveis aleatórias $\quad 56$

Tabela 3.2 - Permutações possíveis usando-se 3 variáveis aleatórias $\quad 57$

Tabela 3.3 - Coeficientes de variação para os parâmetros do solo 65

Tabela 4.1 - Parâmetros do solo $\quad 71$

Tabela 4.2 -Posição dos piezômetros com as respectivas cotas para cada nível do reservatório $\quad 72$

Tabela 4.3 - Resultados das análises $\quad 76$

Tabela 5.1 - Pesos específico dos solos $\quad 85$

Tabela 5.2 - Coesão e ângulo de atrito dos solos obtidos através de ensaios triaxiais e pelo método das combinações de tensões 85

Tabela 5.3 - Coesão e ângulo de atrito dos solos obtidos através de ensaios triaxiais e pelo método dos mínimos quadrados. 86

Tabela 5.4 - Coesão e ângulo de atrito dos solos obtidos através de ensaios de cisalhamento direto e pelo método das combinações de tensões. 86 Tabela 5.5 - Coesão e ângulo de atrito dos solos obtidos através de e nsaios de cisalhamento direto e pelo método dos mínimos quadrados. $\quad 86$ Tabela 5.6 - Estudo comparativo entre $\beta$ e $\beta^{\star} \quad 105$ 


\section{Lista de Símbolos}

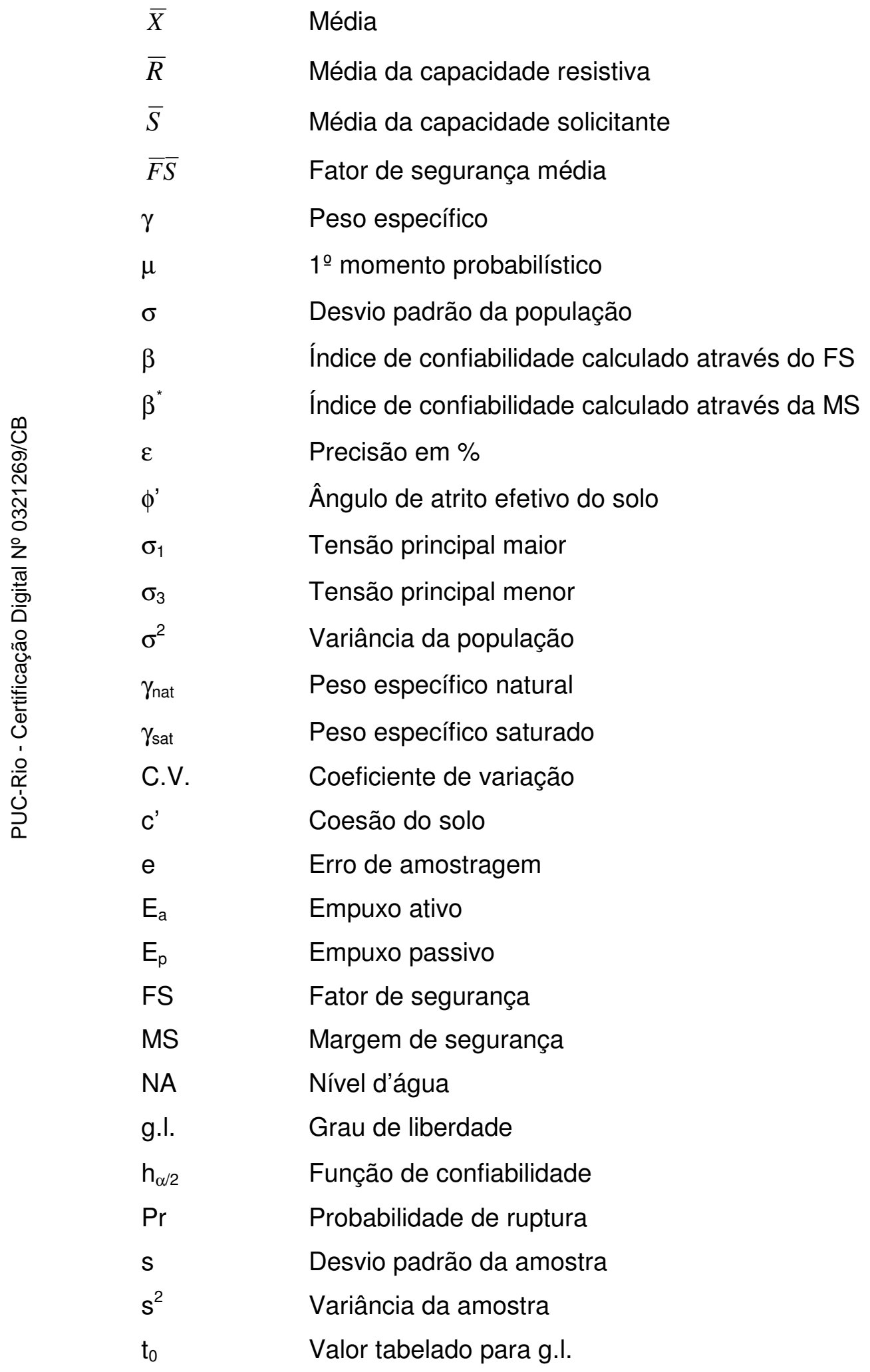




$\begin{array}{ll}u & \text { Poropressão } \\ V & \text { Variância } \\ z & \text { Variável aleatória padronizada } \\ \delta & \text { ângulo de atrito solo/muro } \\ \Phi(\beta) & \text { Curva normal padronizada }\end{array}$

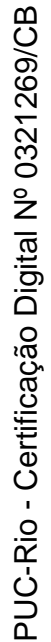

